\title{
Two-Way Clinical Messaging in a CML Specialty Pharmacy Service Model
}

\author{
Christine Sawicki, RPh, MBA, CSSLMBB; Kayla E. Friend, PharmD, BCGP; Ruchik Patel, PharmD; \\ Jennifer M. Polinski, ScD, MPH; and Surya Singh, MD
}

\begin{abstract}
BACKGROUND: Adherence to treatment is correlated with treatment success in chronic myeloid leukemia (CML). CVS Specialty explored novel methods to improve adherence in this population to ensure optimal adherence and lower the risk of unsuccessful treatment. One novel program explored involved an interactive 2-way clinical messaging technology that coaches patients with adherence and knowledge about taking their treatment and managing their conditions. Clinical secure messaging is a 2-way messaging program distinct from the 1-way refill reminders and order status messages that patients were receiving if opted into the messaging program.
\end{abstract}

OBJECTIVE: To assess the effect on adherence of 2-way clinical messaging in a CML population treated with tyrosine kinase inhibitors (TKIs) compared with patients enrolled in 1-way refill reminders.

METHODS: A retrospective cohort study was conducted using prescription claims data. Patients new to TKI therapy and enrolled in at least 1-way messaging were identified and divided into control and study cohorts based on clinical messaging enrollment status. Participants were followed for 365 days after their first fill. Adherence outcomes were defined by medication possession ratio (MPR), length of therapy, first fill drop-off rate, and gap days between refills. Optimal adherence was defined as an $M P R \geq 85 \%$.

RESULTS: Patients receiving clinical messaging had on average a $7.64 \%$ higher MPR score (MPR: $73.94 \%$ vs. $66.30 \%$ ) compared with the control arm $(P=0.0063)$. This translates to $22 \%$ more patients being optimally adherent while exposed to clinical messaging $(P=0.022)$. Patients in the exposed group had a mean 32-day increase in average length of therapy compared with the control group (243 days vs. 275 days, $P=0.0043$ ), potentially driving an increase in adherence. Additional drivers included a 5.4 percentage point reduction in first fill drop-off rates $(4.66 \%$ vs. $10.04 \%$, $P=0.0149$ ). Persistency after 12 months was similar between the study arms (41\%).

CONCLUSIONS: Two-way clinical messaging positively affected adherence outcomes in a CML population. This effect was in addition to 1-way refill reminders and order status messages. The nature of the clinical content encourages further investigation into this novel execution of adherence coaching and counseling through a digital platform.

J Manag Care Spec Pharm. 2019;25(11):1290-96

Copyright $\odot 2019$, Academy of Managed Care Pharmacy. All rights reserved.

\section{What is already known about this subject}

Adherence to tyrosine kinase inhibitor treatment in chronic myeloid leukemia has been associated with treatment success; nonadherence has been tied to treatment failure.

Integrated digital messaging with adherence coaching and counseling has been demonstrated to improve adherence and other health-related behaviors.

One-way text reminders have been shown to improve adherence in patients.

\section{What this study adds}

This retrospective observational cohort analysis calculated the incremental effect that a layered digital clinical messaging program can achieve in a specialty pharmacy subpopulation.

Overall, this program demonstrated statistically significant improvement in adherence and several adherence drivers, such as length of therapy and first fill drop off.

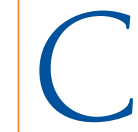

hronic myeloid leukemia (CML), a myeloid cell cancer, affects approximately 1.8 per 100,000 men and women, comprising $0.5 \%$ of all cancer cases within the United States. Patients with CML have a $66.9 \%$ 5-year survival rate. ${ }^{1,2}$ For the most common type of CML, extended or indefinite treatment with tyrosine kinase inhibitors (TKIs) can halt or reduce progression. Gaps in treatment may be correlated with relapse. TKIs used for the treatment of relapsed or remitting disease are defined as second-line TKI therapies. ${ }^{3}$ Nilotinib and bosutinib originally were approved as such second-line therapies ${ }^{4,5}$; for the purpose of this study, these agents will be defined as second-line agents. Studies have confirmed a correlation between TKI nonadherence and suboptimal changes in clinical measurements of treatment efficacy, including cytogenic response rate and mean molecular response rate, and between nonadherence and increased resource utilization costs. ${ }^{6-11}$ Increased TKI adherence likely decreases the chances of disease progression or relapse in CML in first-line and second-line treatments.

Patient participation in health coaching has been associated with improvements in medication adherence and other health behaviors. ${ }^{12-16}$ Health coaching centers on education and 
Two-Way Clinical Messaging in a CML Specialty Pharmacy Service Model

\section{TABLE 1 Baseline Characteristics}

\begin{tabular}{|c|c|c|c|}
\hline & $\begin{array}{l}\begin{array}{c}\text { Clinical Messaging } \\
\mathrm{n}=279\end{array} \\
\text { ? }\end{array}$ & $\begin{array}{c}\text { Matched Controls } \\
\mathbf{n}=279\end{array}$ & $P$ Value \\
\hline \multicolumn{4}{|l|}{ Patient demographics } \\
\hline Male, \% (n) & $52.0 \quad(145)$ & $54.8 \quad(153)$ & 0.50 \\
\hline Age at first fill, mean \pm SD & $53.3 \pm 13.3$ & $54.4 \pm 14.5$ & 0.35 \\
\hline \multicolumn{4}{|l|}{ Census data (using patient ZIP code) } \\
\hline Median income, mean \pm SD & $\$ 63,683 \pm \$ 25,820$ & $\$ 64,182 \pm \$ 25,107$ & 0.82 \\
\hline$\%$ with college degree, mean \pm SD & $19.3 \pm 9.2$ & $20.0 \pm 9.2$ & 0.34 \\
\hline$\%$ poverty, mean \pm SD & $11.9 \pm 7.5$ & $12.0 \pm 8.1$ & 0.88 \\
\hline$\%$ African American, mean \pm SD & $12.7 \pm 16.9$ & $13.7 \pm 20.3$ & 0.54 \\
\hline \multicolumn{4}{|l|}{ Drug, \% (n) } \\
\hline Imatinib & $47.3(132)$ & $47.3(132)$ & \multirow{4}{*}{$\begin{array}{l}\text { N/A (exact match on } \\
\text { drug at first fill) }\end{array}$} \\
\hline Dasatinib & $38.0(106)$ & $38.0(106)$ & \\
\hline Nilotinib & $12.5 \quad(35)$ & $12.5 \quad(35)$ & \\
\hline Bosutinib & $2.2 \quad(6)$ & $2.2 \quad(6)$ & \\
\hline
\end{tabular}

self-efficacy, equipping patients with information and knowledge, thereby cultivating interest and expanding patient involvement in the management of the condition. Increased frequency of patient touch points with a coach is associated with improved medication adherence and patients' health literacy, an important factor that can limit meaningful involvement in their health care decisions. ${ }^{17}$ Digital messaging offers an attractive venue for health care coaching interactions.

The average adult in the United States checks his/her mobile device an average of 80 times a day. ${ }^{18}$ Given the frequency of these mobile device interactions, incorporating adherencerelated messages into texts to patients (1-way texting) has been associated with improved adherence. 19,20

In 2016, CVS Specialty implemented 2-way clinical messaging technology (provider messages the patient, patient can in turn message the provider). Patients receive personalized messages based on their specific clinical and medication profiles. With receipt of each message, patients have the opportunity to ask questions and communicate difficulties or successes. We hypothesized that using discreet clinical messages would enhance communication and would be more effective than l-way texting in improving adherence to TKIs among CML patients.

\section{Methods}

\section{The Intervention: 2-Way Clinical Messaging}

As part of usual care, CVS Specialty patients have the opportunity to set preferences to receive digital (text or email) or telephonic communication services. Patients who prefer digital communications can receive a text message with prescription status (e.g., prescription status, refill reminders, and shipment tracking). Texts may be sent in addition to, or as a replacement for, traditional phone calls. Patients participating in the 1-way texting service may opt for refill reminders, status updates, or both. Each option sends an average of 1 text per month.

Recently, to improve its patient care experience, CVS Specialty developed a clinical messaging program designed to deliver an even higher level of digital support to all patients, in addition to the l-way texting service. The clinical messaging program, available to all specialty patients, differs from 1-way texting in 2 important ways: the breadth of topics covered and the bidirectional nature of the interactions.

First, clinical messaging has a number of additional topic categories, such as lab testing, adherence to prescribed therapy, symptoms and side effects, and condition-specific management guidance. The messages are developed by pharmacists and vetted through a multidisciplinary clinical review committee. Then the messages undergo a quality check on a regular basis to remain current with evidence-based information. Pertinent messages are shared with patients at the points in time when they are most appropriate, for example, counseling messages for taking imatinib with food and plenty of water (to avoid gastrointestinal upset) or lab monitoring message at 3 months of therapy (when the response to therapy should be assessed).

Second, to support coaching, messages include a hyperlink so that patients can request additional consultation with a specially trained pharmacist- $5 \%$ of patients requested this feature. Some messages also prompt patients to respond via text to a specific question (e.g., "REPLY $1=$ All set, no questions, $2=$ ASK a question; REPLY $1=$ test scheduled, 2 =planning to schedule soon; REPLY 1 =test done, 2 =test scheduled soon, $3=$ no test planned"). These messages are sent to patients at 3, 6, 9, and 12 months in accordance with recommended safety monitoringwe saw over $40 \%$ response rate for these messages. 


\section{TABLE 2 Study Results}

\begin{tabular}{|c|c|c|c|c|}
\hline & $\begin{array}{c}\text { Matched Controls } \\
n=279\end{array}$ & 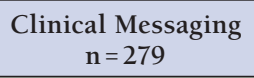 & Delta & $P$ Value \\
\hline \multicolumn{5}{|l|}{ All therapies } \\
\hline MPR & 66.3 & 73.9 & 7.6 & 0.01 \\
\hline Optimally adherent, \% (n) & $43.7(122)$ & $53.4(149)$ & $9.7 \%$ & 0.02 \\
\hline Length of therapy, days & 242.9 & 274.9 & 32.0 & 0.01 \\
\hline First fill drop-off, \% (n) & $10.0(28)$ & $4.7(13)$ & $-5.4 \%$ & 0.02 \\
\hline Average gap days & 7.8 & 8.0 & 0.2 & 0.91 \\
\hline \multicolumn{5}{|c|}{ First-line therapies only (imatinib, dasatinib) } \\
\hline MPR & 67.6 & 75.5 & 7.8 & 0.01 \\
\hline Optimally adherent, \% (n) & $45.4(126)$ & $54.6(152)$ & $9.2 \%$ & 0.04 \\
\hline Average length of therapy, days & 245.3 & 279.5 & 34.2 & 0.01 \\
\hline First fill drop-off, \% (n) & $9.7(27)$ & $3.8(11)$ & $-5.9 \%$ & 0.01 \\
\hline Average gap days & 7.5 & 7.8 & 0.3 & 0.85 \\
\hline Imatinib MPR $(\mathrm{n}=212)$ & 69.4 & 73.9 & $4.5 \%$ & 0.28 \\
\hline Dasatinib MPR $(n=212)$ & 65.5 & 76.0 & $10.5 \%$ & 0.01 \\
\hline Nilotinib MPR $(\mathrm{n}=70)$ & 60.5 & 65.9 & $5.4 \%$ & 0.53 \\
\hline Bosutinib MPR $(\mathrm{n}=12)$ & 46.9 & 60.4 & $13.5 \%$ & 0.53 \\
\hline
\end{tabular}

During our pilot study, an average 1.5 messages were sent per month. CVS Specialty patient service representatives (PSR) reviewed all patient responses. If a patient texted back a question, the pharmacist provided coaching and follow-up with the patient by phone. In addition, either the pharmacist or a PSR contacted the patient's prescriber in the following instances: no response to a specific question, 2 consecutive lab-monitoring responses of either "no response" or "no test planned," and a change in therapy.

\section{Study Design and Patient Eligibility}

Given the hypothesized improvement in patient experience attributable to the enhanced clinical messaging program, we conducted a retrospective cohort study. Patients who were initiated on TKI therapy (imatinib mesylate, dasatinib, bosutinib, or nilotinib) between February 1, 2016, and December 15, 2016, were eligible for this study. PSRs offered the program to patients during their enrollment calls to CVS Specialty for a first prescription for one of these therapies. Patients who could not be reached via phone by PSRs but were enrolled in l-way texting were assigned to the control group. To address potential differences in levels of motivation between patients, we required all study patients to be at least enrolled in 1-way texting. Patients who were reached and declined 1-way texting or clinical messaging were excluded from the study. Each study participant was followed for a period of 365 days, starting on the date of the first TKI medication fill date.

\section{Data Sources and Outcomes}

Patient demographic and prescription drug utilization data were obtained from the CVS Specialty claims database. Oneway texting and clinical messaging enrollment data were obtained from messaging program enrollment files and the pharmacy patient profile.

Medication adherence was calculated as the medication possession ratio (MPR) during the 365 days of follow-up: total days supply $\div 365$ days $\times 100$, truncated at $100 \%$. Optimally adherent patients were defined as having had an MPR of $>85 \%$. This threshold is based on studies demonstrating differences in cytogenic response rates and mean molecular responses between patients with CML at and below 85\% MPR. ${ }^{11}$ Adherence drivers recorded and analyzed included length of therapy (time between initiating therapy and the exhaust date of the last fill); first fill drop-off (discontinuation of therapy after 1 fill and no further fills during the follow-up period); and gap days between refills (among patients with at least 2 prescription fills, average gap days were summed between exhaust dates of previous fills and next fill dates, divided by the number of refills).

Medication persistency was measured as a secondary outcome. A gap in medication persistency was defined as a period of >60-day gap between fills (switching among TKI therapies was allowed here, so that patients who switched were not included as nonpersistent). To examine persistency variances between first- and second-line therapies, we looked at persistence by drug.

\section{Covariates}

To mitigate differences in demographic and prescription drug use before follow-up between clinical messaging and control 


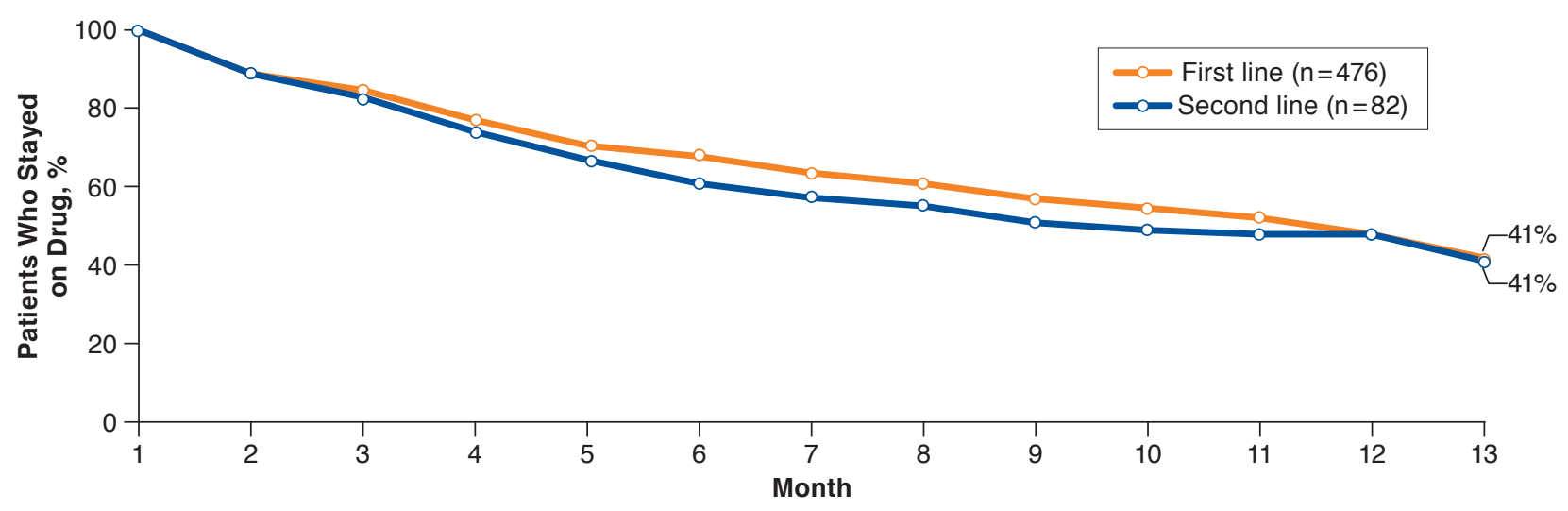

patients, each participant in clinical messaging was matched to a control patient initiating the same drug via propensity score greedy matching. The propensity score model included the following covariates: age, gender, index fill date, and additional census-based data in the patient's ZIP code of residence, such as median household income, percentage with a college degree, percentage at or below the poverty level, and percentage of African-American race.

\section{Statistical Analyses}

Baseline characteristics were compared between the control and clinical messaging groups using descriptive analyses. Outcomes between the matched groups were assessed using 2-sample t-tests and chi-square tests. We also calculated risk differences and the number needed to treat. Here, the number needed to treat is the number of patients needing clinical messaging for 1 more patient to have optimal adherence. Therapy persistence for all drugs and each drug was compared between the clinical messaging and control groups using Cox proportional hazards models. We also produced corresponding Kaplan-Meier curves.

\section{Results}

After 1:1 propensity score matching, 279 patients were assigned in each group, which included $81 \%$ of the population. Imatinib $(n=238)$ and dasatinib $(n=212)$ were the most frequently used agents, followed by nilotinib $(n=70)$ and bosutinib $(n=12)$. Baseline characteristics were balanced between the groups (Table 1). For example, gender $(52.0 \%$ male, $54.8 \%$ male, $P=0.50)$ and age at first fill (53.3 years, 54.4 years, $P=0.35$ ) were similar between the matched intervention and control groups.

Overall, enrollment and participation in clinical messaging was associated with improved adherence to medication. MPR for participants in clinical messaging was $73.9 \%$, which was 7.6 percentage points higher than the control group's MPR of $66.3 \%(P=0.01$; Table 2). At the drug level, the dasatinib group was the only one to achieve statistically significant differences between the compared arms with a difference of $10.5 \%(P=0.01)$. Of the clinical messaging patients, $53.4 \%$ were optimally adherent, compared with $43.7 \%$ in the control group, a difference of 9.7 percentage points $(P=0.02)$. Clinical messaging patients were $22 \%$ more likely to be optimally adherent. The number needed to treat for an additional patient to be optimally adherent was 17 (data not shown).

Among all patients, the average length of therapy was 243 days for the control group and 275 days for the intervention group, an increase of 32 days $(P=0.01$; Table 2). The first fill drop-off rate was $10.0 \%$ in the control group versus $4.7 \%$ in the exposed group, a difference of 5.4 percentage points $(P=0.02)$. Average gap days for the clinical messaging and control groups were similar. Persistency was similar between firstand second-line drugs, with yet only $41 \%$ of patients remaining on therapy after 12 months (Figure 1). Compared with the control group, intervention group patients were more likely to persist on first-line therapy (hazard ratio $[\mathrm{HR}]=1.2$, 95\% confidence interval $[\mathrm{CI}]=0.82-1.74$; Figure 2 ). The effect was similar for second-line therapies, with intervention patients 1.8 (95\% $\mathrm{CI}=0.75-4.35)$ times as likely to persist on therapy compared with controls (Figure 3).

\section{Discussion}

In this study, we evaluated the effect of clinical messaging on adherence to CML therapies. Patients participating in the program realized a 7.6 percentage point improvement in MPR compared with patients not exposed to clinical messaging. This corresponds to a $22 \%$ greater likelihood of optimal adherence among clinical messaging patients. Persistence to therapy was 


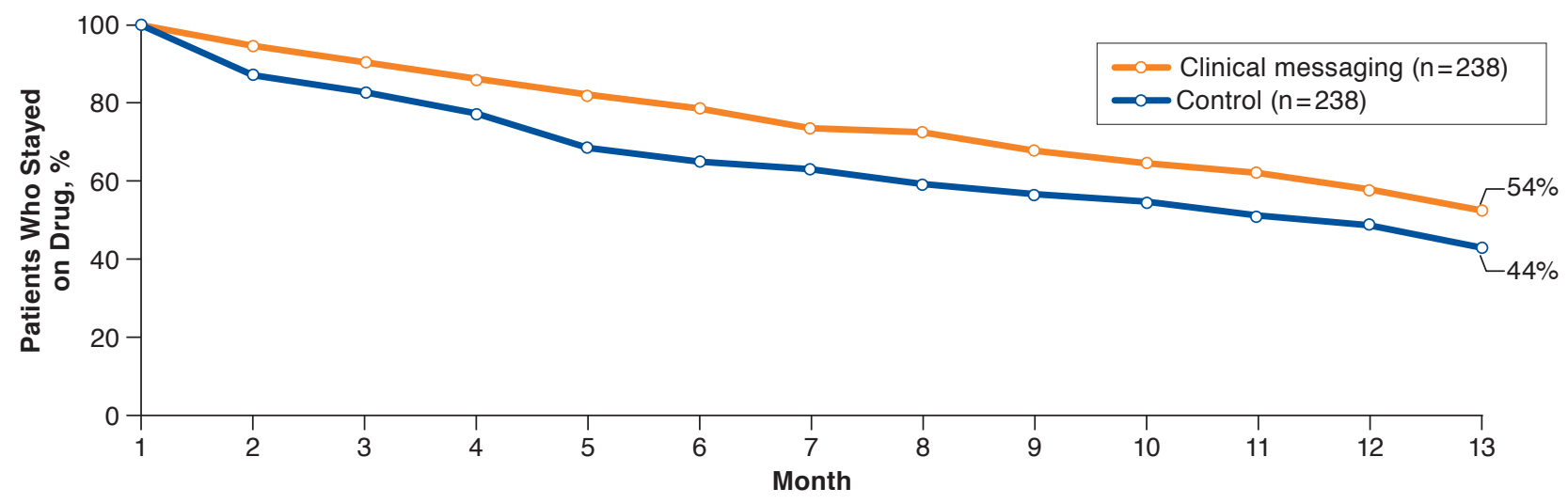

no different between first- and second-line therapies, with only $41 \%$ of patients still taking their medications at 12 months. Clinical messaging participants were significantly more persistent to therapy over time when taking imatinib or dasatinib compared with controls, and for every 17 patients who receive clinical messaging, 1 patient achieved optimal adherence during the follow-up. Because of the lack of literature on the potential adherence effect of a 2-way adherence coaching program in this or other clinical scenarios, contextualizing these outcomes is difficult. Condition management programs using clinical messaging to communicate care-specific information and adherence coaching have been studied in diabetes mellitus, cardiovascular disease, and chronic lung disease, with 16 of 41 studies reporting significant improvements in clinical outcomes. ${ }^{20}$

This review attempts to define the value of clinical messaging; however, due to the study design, we cannot directly compare the interventions because the control group was receiving refill reminders and order status messaging from the dispensing pharmacy. Given this study's finding of an adherence increase, there is likely added incremental value derived from the content and delivery of these enhanced 2-way clinical messages. However, less than $60 \%$ of study patients remained on therapy at the end of the 12-month study period, suggesting that additional care services and programs are warranted in this population to achieve sustained therapy adherence.

An additional factor to consider in why patients discontinued therapy is the discontinuance of therapy by the provider because of intolerance or lack of efficacy, which was not measured in our study. One improvement suggested by study participants may be to deliver the clinical messages within a 2-way HIPAA-compliant secure digital tool. This enhancement has the potential to positively influence patient engagement, adherence, and persistence.

\section{Limitations}

This study has some limitations to consider. Patient interactions with the clinical messaging program were tracked separately from and, with no linking identifier to, the patients' prescription drug claims. Without this linkage, we could not have determined what proportion of the observed adherence improvements were attributable to dialogue initiated by patients in response to 2-way texts and what proportion was attributable to the information provided in the messages, which were more frequent and covered a broader scope of topics.

This study was not randomized, so an imbalance of baseline characteristics between the clinical messaging and control groups is likely. We used propensity score matching to mitigate observable differences between the groups. Imbalances in patient characteristics that we could not measure may remain. In particular, patients who signed up for clinical messaging may be more motivated to take care of their health than patients in the control group. To minimize this potential motivation bias, we required participants and control patients to be enrolled in the 1-way texting program.

\section{Conclusions}

Clinical messaging was associated with improved adherence in a population of patients receiving first- and second-line therapies for CML. Our results suggest that a combination of clinical messaging and adherence reminders delivered digitally are associated with adherence improvements. Given the increasing ubiquity of digital tools, particularly those related to health, the potential for convenient, real-time health interactions with a clinical provider to influence and optimize patient health behaviors is high. Based on the initial findings in this evaluation, CVS Specialty is continuing to develop new technologies to offer messaging to all specialty patients for a variety of specialty conditions. 


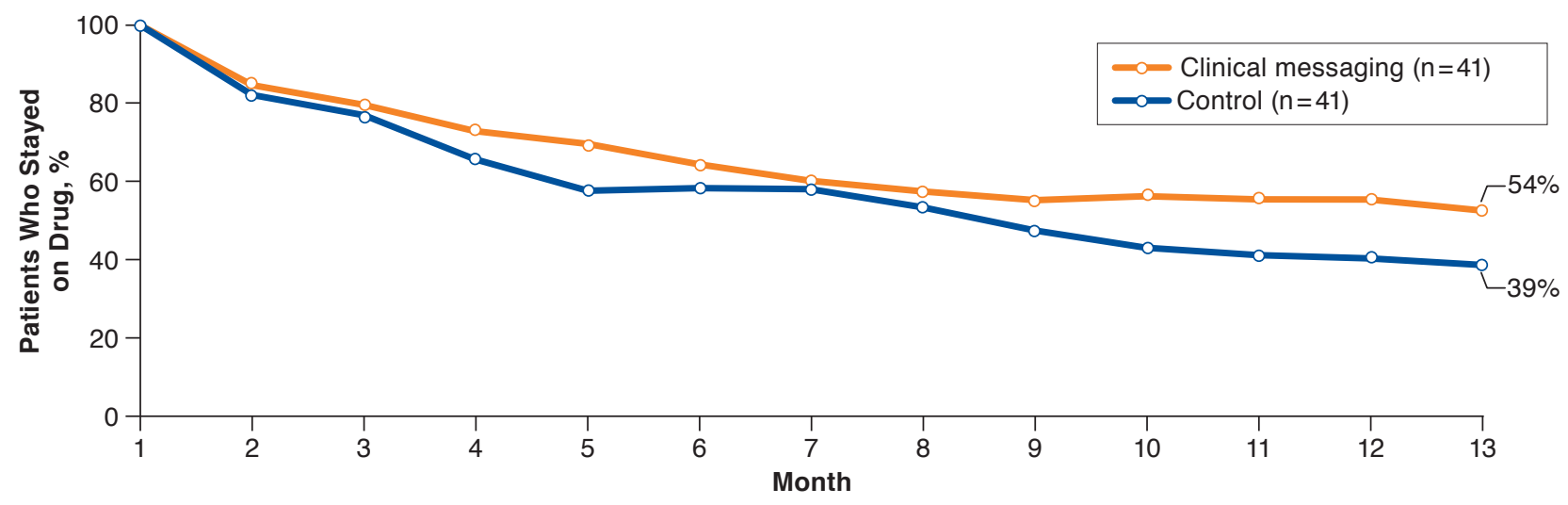

Because the program offers additional opportunities for patient and health care provider communication outside of typical medical visits and pharmacy visits, we may see parallels to the history of telemedicine and its eventual recognition and reimbursement, with digitally delivered health care coaching and adherence counseling becoming a reimbursable pharmacist-led service. ${ }^{21}$ In the ongoing effort to optimize the clinical benefit that patients receive from the medications they are prescribed, digital interventions such as the one described here represent a sizable opportunity for improvement.

\section{Authors}

CHRISTINE SAWICKI, RPh, MBA, CSSLMBB, and KAYLA E. FRIEND, PharmD, BCGP, Specialty Clinical Innovation and Product Development, CVS Health, Lincoln, Rhode Island; RUCHIK PATEL, PharmD, Specialty Clinical Innovation and Program Development, CVS Health, Lincoln, Rhode Island; JENNIFER M. POLINSKI, ScD, $\mathrm{MPH}$, Enterprise Evaluation and Population Health Analytics, CVS Health, Woonsocket, Rhode Island; and SURYA SINGH, MD, Vice President and Specialty Chief Medical Officer, CVS Health, Lincoln, Rhode Island.

AUTHOR CORRESPONDENCE: Christine Sawicki, RPh, MBA, CSSLMBB, Director, Specialty Clinical Innovation and Product Development, CVS Health, 695 George Washington Hwy., Lincoln, RI 02865. Tel.: 401.741.0303; E-mail: Christine.Sawicki@CVSHealth.com.

\section{DISCLOSURES}

Funding for this research was provided by CVS Health. The sponsor was involved at all stages of the study's conduct and reporting. Sawicki and Friend are employed by CVS Health. The other authors were employed by CVS at the time of this study. The authors have nothing more to disclose.
Posters based on this work were presented at the AMCP Managed Care \& Specialty Pharmacy Annual Meeting 2016; April 19-22, 2016; San Francisco, CA, and AMCP Nexus 2016; October 3-6, 2016; National Harbor, MD.

\section{REFERENCES}

1. Kiel PJ, Fausel CA. Chronic leukemias. In: DiPiro JT, Talbert RL, Yee GC, eds. Pharmacotherapy: A Pathophysiologic Approach. 10 ed. New York: McGraw-Hill; 2017.

2. Jemal A, Ward EM, Johnson CJ, et al. Annual report to the nation on the status of cancer, 1975-2014, featuring survival. J Natl Cancer Inst. 2017;109(9):djx030. Available at: https://www.ncbi.nlm.nih.gov/pmc/articles/ PMC5409140/. Accessed September 29, 2019

3. O'Brien S, Radich JP, Abboud CN, et al. Chronic myelogenous leukemia, version 1.2014. J Natl Compr Canc Netw. 2013;11(11):1327-40. Available at: https://www.ncbi.nlm.nih.gov/pmc/articles/PMC4234105/. Accessed September 29, 2019.

4. Tasigna (nilotinib) capsules, for oral use. Novartis. March 2018. Available at: https://www.pharma.us.novartis.com/sites/www.pharma.us.novartis. com/files/tasigna.pdf. Accessed September 29, 2019.

5. Bosulif (bosutinib) monohydrate tablet, film coated. Pfizer. August 2019. Available at: http://labeling.pfizer.com/ShowLabeling.aspx?id=884. Accessed September 29, 2019.

6. Noens L, Van lierde MA, De bock R, et al. Prevalence, determinants, and outcomes of nonadherence to imatinib therapy in patients with chronic myeloid leukemia: the ADAGIO study. Blood. 2009; 113(22):5401-11. Available at: http://www.bloodjournal.org/content/113/22/5401.long?ssochecked=true. Accessed September 29, 2019.

7. Darkow T, Henk HJ, Thomas SK, et al. Treatment interruptions and nonadherence with imatinib and associated healthcare costs: a retrospective analysis among managed care patients with chronic myelogenous leukaemia. Pharmacoeconomics. 2007;25(6):481-96.

8. Halpern R, Barghout V, Zarotsky V, Williams D. Costs and utilization associated with imatinib adherence in patients with chronic myeloid leukemia or gastrointestinal stromal tumors. J Clin Outcomes Manag. 2009;16(5):215-23. Available at: https://pdfs.semanticscholar.org/4123/099a 2310cd6ab0b0763b9103cf742d0e6d33.pdf. Accessed September 29, 2019.

9. Wu EQ, Johnson S, Beaulieu N, et al. Healthcare resource utilization and costs associated with non-adherence to imatinib treatment in chronic myeloid leukemia patients. Curr Med Res Opin. 2010;26(1):61-69. 
10. Noens L, Hensen M, Kucmin-Belelmans I, Lofgren C, Gilloteau I, Vrijens B. Measurement of adherence to BCR-ABL inhibitor therapy in chronic myeloid leukemia: current situation and future challenges. Haematologica. 2014;99(3):437-47. Available at: https://www.ncbi.nlm.nih.gov/pmc/articles/ PMC3943306/. Accessed September 29, 2019.

11. Marin D, Bazeos A, Mahon FX, et al. Adherence is the critical factor for achieving molecular responses in patients with chronic myeloid leukemia who achieve complete cytogenetic responses on imatinib. J Clin Oncol. 2010;28(14):2381-88.

12. Klein M, Mogles N, van Wissen A. Intelligent mobile support for therapy adherence and behavior change. J Biomed Inform. 2014;51:137-51. Available at: https://www.sciencedirect.com/science/article/pii/ S1532046414001221?via\%3Dihub. Accessed September 29, 2019.

13. Schneider SM, Adams DB, Gosselin T. A tailored nurse coaching intervention for oral chemotherapy adherence. J Adv Pract Oncol. 2014;5(3):16372. Available at: https://www.ncbi.nlm.nih.gov/pmc/articles/PMC4114492/. Accessed September 29, 2019.

14. Thom DH, Willard-Grace R, Hessler D, et al. The impact of health coaching on medication adherence in patients with poorly controlled diabetes, hypertension, and/or hyperlipidemia: a randomized controlled trial. J Am Board Fam Med. 2015;28(1):38-45. Available at: http://www.jabfm.org/ content/28/1/38.full.pdf+html. Accessed September 29, 2019.
15. Zullig LL, Peterson ED, Bosworth HB. Ingredients of successful interventions to improve medication adherence. JAMA. 2013;310(24):2611-12.

16. Osterberg L, Blaschke T. Adherence to medication. N Engl J Med. 2005;353(5):487-97.

17. Adams RJ. Improving health outcomes with better patient understanding and education. Risk Manag Healthc Policy. 2010;3:61-72. Available at: https://www.ncbi. nlm.nih.gov/pmc/articles/PMC3270921/. Accessed September 29, 2019.

18. Americans check their phones 80 times a day: study. New York Post. November 8, 2017. Available at: https://nypost.com/2017/11/08/americanscheck-their-phones-80-times-a-day-study/. Accessed September 29, 2019.

19. Birnbaum F, Lewis D, Rosen RK, Ranney ML. Patient engagement and the design of digital health. Acad Emerg Med. 2015;22(6):754-56. Available at: https://onlinelibrary.wiley.com/doi/full/10.1111/acem.12692. Accessed September 29, 2019.

20. Hamine S, Gerth-Guyette E, Faulx D, Green BB, Ginsburg AS. Impact of mHealth chronic disease management on treatment adherence and patient outcomes: a systematic review. J Med Internet Res. 2015;17:e52. Available at: https://www.ncbi.nlm.nih.gov/pmc/articles/PMC4376208/. Accessed September 29, 2019.

21. Littauer SL, Dixon DL, Mishra VK, Sisson EM, Salgado TM. Pharmacists providing care in the outpatient setting through telemedicine models: a narrative review. Pharm Pract (Granada). 2017;15(4):1134. Available at: https://www. ncbi.nlm.nih.gov/pmc/articles/PMC5742004/. Accessed September 29, 2019. 